\title{
Sociodemographic predictors of elderly's psychological well-being in Malaysia
}

\begin{abstract}
Background and objective: Psychological well-being as one of the most important indicators of successful aging has received substantial attention in the gerontological literature. Prior studies show that sociodemographic factors influencing elderly's psychological well-being are multiple and differ across cultures. The aim of this study was to identify significant sociodemographic predictors of psychological well-being among Malay elders. Methodology: The study included 1415 older Malays (60-100 years, 722 women), randomly selected through a multistage stratified random method from Peninsular Malaysia. WHO-Five wellbeing index was used to measure psychological well-being. Data analysis was conducted using the Statistical Package for Social Sciences (SPSS) version 13.0. Results: Using multiple regression analysis a significant model emerged $(F(7,1407)=20.14, p \leq 0.001)$, where age, sex, marital status, and household income were significant predictor variables of psychological well-being among Malay elders. However, level of education, employment status, and place of residence failed to predict psychological well-being. Conclusion: This study showed that the oldest old, elderly women, unmarried, and the poor elderly people are at risk for experiencing low psychological well-being. Therefore, they need special attention from family, policy makers, and those who work with elderly people.
\end{abstract}

Keyword: Psychological well-being; Socioeconomic status; Sex; Older Malaysians; Multiple regression analysis 